\title{
IRREGULARIDADES MEIÓTICAS EN HÍBRIDOS INTERGENÉRICOS Helianthus annuus L. x Tithonia rotundifolia (Mill.) S.F. Blake
}

\author{
MEIOTIC IRREGULARITIES IN INTERGENERIC HYBRIDS \\ Helianthus annuus L. x Tithonia rotundifolia (Mill.) S.F. Blake
}

\author{
Martha Gómez-Martínez y M. Humberto Reyes-Valdés*
}

Departamento de Fitomejoramiento, Universidad Autónoma Agraria Antonio Narro. Calzada Antonio Narro 1923. 25315, Saltillo, Coah., México. Tel. (844)411-0296.

*Autor para correspondencia: MathGenome@gmail.com

\section{RESUMEN}

El girasol cultivado, Helianthus annuus, y la especie silvestre Tithonia rotundifolia, son miembros de dos géneros pertenecientes a la familia de las asteráceas y comparten un número cromosómico común $(2 n=2 x=$ 34). En esta investigación se analizaron los apareamientos cromosómicos meióticos de híbridos intergenéricos $H$. annuus $x T$. rotundifolia, obtenidos en una investigación previa, en la cual todas las plantas resultaron estériles. La técnica de preparación de células meióticas consistió en aplastamiento y tinción con colorante carmín. Los meiocitos en diacinesis, metafase I y anafase I, se analizaron en un microscopio compuesto. La meiosis en los híbridos F1 presentó apareamientos altamente irregulares, con la presencia de univalentes y multivalentes en diacinesis-metafase I, cromosomas adelantados, cromosomas rezagados y puentes cromosómicos en anafase $\mathrm{I}$, así como díadas desbalanceadas al final de la anafase I. El apareamiento anormal indica que hay una segregación cromosómica irregular que está originando la esterilidad tanto masculina como femenina previamente reportada de los híbridos intergenéricos. Se infiere que los genomas parentales (Línea HA 89 de $H$. annuus y $T$. rotundifolia Ac. 26) son incompatibles en la meiosis, lo cual ocasiona una segregación irregular que impide la formación de gametos viables, y deshabilita la capacidad reproductiva sexual de las plantas híbridas.

Palabras clave: Asteraceae, apareamiento meiótico, hibridación intergenérica, segregación cromosómica irregular.

\section{SUMMARY}

Cultivated sunflower, Helianthus annuus, and wild species, Tithonia rotundifolia, are members of the Asteraceae family and share a common chromosome number $(2 n=2 x=34)$. In this research, meiotic chromosome pairing was analyzed in $H$. annuus $x T$. rotundifolia intergeneric hybrids obtained in previous research, where all plants were sterile. The squash technique with carmine staining was used for meiotic cell preparation. The meiocytes in diakinesis, metaphase I and anaphase I were analyzed under a compound microscope. Meiosis in F1 hybrids showed highly irregular pairing, with presence of univalent and multivalent configurations, fast chromosomes, lagged chromosomes and bridges in anaphase I, as well as unbalanced dyads at the end of anaphase I. The observed abnormal pairing indicates irregular chromosome segregation causing male and female sterility, as previously in the intergeneric hybrids. It is inferred that parental genomes (HA 89 strain of $H$. annuus and $T$. rotundifolia Ac. 26) are incompatible in meiosis, causing unbalanced segregation that precludes viable gamete formation and impairs sexual reproductive capacity of hybrid plants.

Index words: Asteraceae, meiotic pairing, intergeneric hybridization, irregular chromosome segregation.

\section{INTRODUCCIÓN}

La hibridación interespecífica de plantas se ha utilizado en investigación básica y aplicada de fenómenos biológicos. Este tipo de hibridación es importante en el mejoramiento genético, como una estrategia para transferir genes deseables de las especies silvestres a las plantas cultivadas. Asimismo, los experimentos de hibridación amplia ayudan a dilucidar relaciones filogenéticas y divergencia cromosómica.

Una especie que podría utilizarse como fuente de genes para el girasol cultivado (Helianthus annuus) a través de hibridación es Tithonia rotundifolia, la cual es una planta anual monocárpica de reproducción sexual (Muoghalu y Chuba, 2005) que abunda en varias regiones tropicales de México (Reyes-Valdés et al., 2007). Junto con Helianthus, forma parte del conjunto Viguiera y pertenecen a la familia de las asteráceas, que comparten, entre otros rasgos, el número cromosómico básico común $2 n=2 x=34$ (Seiler y Rieseberg, 1997). T. rotundifolia posee características ornamentales deseables para ser transferidas a variedades cultivadas de $H$. annuus: su tallo presenta pubescencia suave y pedicelo largo, con pétalos de atractivo color naranja. Sin embargo, esta especie carece de algunas características que exhibe el girasol cultivado: resistencia a heladas, insensibilidad al fotoperíodo y capítulos grandes.

Hay evidencia de hibridación natural entre especies de Tithonia (Tovar-Sánchez et al., 2012), pero solamente existen tres reportes de su cruzamiento con $\mathrm{H}$. annuus (Cristov y Panayotov, 1991, Reyes-Valdés et al., 2005 y Luévanos-Escareño et al., 2010). Cristov y Panayotov (1991) realizaron cruzamientos entre $H$. annuus y las especies $T$. rotundifolia, $T$. speciosa y $T$. tagetiflora, en los que la variedad Peredovik, y las líneas 3004 y HA 89 fungieron como progenitores femeninos ( $H$. annuus). De los cruzamientos Peredovik x T. rotundifolia y 3004 x T. rotundifolia obtuvieron plantas con una sola inflorescencia, 
mientras que en HA $89 \times$ T. rotundifolia observaron plantas ramificadas. Estos autores afirman haber detectado fertilidad en la progenie de estos cruzamientos, especialmente cuando se utilizó la línea androestéril HA 89 como progenitor femenino. En un reporte posterior (Christov, 2013) se señala que solamente los híbridos $H$. annuus $\times$ T. speciosa alcanzaron la sexta generación.

Los primeros híbridos intergenéricos $H$. annuus $\times$ T. rotundifolia confirmados con huella genética molecular fueron desarrollados por Reyes-Valdés et al. (2005), quienes obtuvieron tres aquenios del cruzamiento de 20 plantas androestériles de la línea HA 89 polinizadas con T. rotundifolia. El híbrido presentó una sola cabezuela como $\mathrm{H}$. annuus cultivado, su inflorescencia fue parecida a la de $T$. rotundifolia, y no produjo descendencia.

Posteriormente, Luévanos-Escareño et al. (2010) desarrollaron híbridos intergenéricos del cruzamiento entre la línea androestéril HA 89 y una población de T. rotundifolia (Ac. 26) colectada en Iguala, Guerrero, México. Los análisis moleculares con AFLPs, demostraron la naturaleza híbrida de los individuos, los cuales fueron estériles ya que no produjeron polen ni semilla.

Los reportes sobre cruzas interespecíficas dentro del género Helianthus son numerosos. Tal es el caso de los híbridos de $H$. annuus con $H$. tuberosus, $H$. salcifolius y Verbisina helianthoides, donde se demostró la utilidad de la hibridación amplia en el mejoramiento del girasol cultivado (Encheva et al., 2014). Jan (1997) efectuó una recopilación amplia de resultados de hibridación interespecífica dentro de la sección Helianthus.

La incidencia de irregularidades cromosómicas varía entre los cruzamientos. Atlagic et al. $(1993,1995)$ hicieron estudios citológicos con híbridos de cruzas de $H$. annuus con $\mathrm{H}$. tuberosus, $\mathrm{H}$. mollis, $\mathrm{H}$. salicifolius y $\mathrm{H}$. maximiliani. Estos autores encontraron meiosis irregulares, con univalentes y multivalentes en diacinesis, cromosomas adelantados en metafase I y cromosomas formando puentes y rezagados en anafase I. Dolgova et al. (2007) observaron una baja frecuencia de irregularidades cromosómicas en cruzas del girasol cultivado con H. argophyllus (6.5 a 8.6 $\%$ ) en comparación con las del girasol con H. praecox que presentó $50 \%$ de aberraciones: univalentes y multivalentes en diacinesis, cromosomas rezagados en metafase I, anafase I y metafase II, así como puentes en anafase I y metafase II.

Los estudios cromosómicos ayudan a esclarecer las relaciones filogenéticas y taxonómicas, han hecho valiosos aportes al conocimiento de los mecanismos de aislamiento reproductivo y modos de especiación en plantas.
En particular, el estudio del comportamiento meiótico les permite determinar el grado de homología cromosómica entre especies y seleccionar los progenitores con mayor probabilidad de compatibilidad en cruzas interespecíficas.

Los materiales de $\mathrm{H}$. annuus y $\mathrm{T}$. rotundifolia utilizados por Luévanos-Escareño et al. (2010) presentan suficiente compatibilidad en combinación híbrida, pero su descendencia es estéril, tanto para la producción de polen funcional como para la formación de semilla. La falta de reproducción sexual en los híbridos interespecíficos puede ser ocasionada por anormalidades cromosómicas meióticas (Baack et al., 2015). Por ello, el presente trabajo tuvo como objetivo evaluar el apareamiento cromosómico meiótico de los híbridos intergenéricos desarrollados por LuévanosEscareño et al. (2010), para averiguar la causa de su infertilidad.

\section{MATERIALES Y MÉTODOS}

Se utilizaron 210 semillas procedentes de los cruzamientos entre la línea androestéril HA 89 ( $H$. annuus) y $T$. rotundifolia (Ac. 26, Iguala, Guerrero) realizados por Luévanos-Escareño et al. (2010). Las semillas se sembraron en vasos de polipropileno que posteriormente se trasladaron a un invernadero de alta tecnología en Saltillo, Coahuila. El trasplante se efectuó en el Campo Experimental de la Universidad Autónoma Agraria Antonio Narro (25 21' LN, $101^{\circ}$ 02 ' LO, a una altitud de $1800 \mathrm{~m}$ ), a una distancia de $50 \mathrm{~cm}$ entre plantas y $80 \mathrm{~cm}$ entre surcos. Solamente 49 plantas alcanzaron la etapa de floración.

Se recolectaron 36 botones florales de los híbridos intergenéricos; los cuales se fijaron en alcohol de $96^{\circ}$ y ácido acético glacial (3:1 v/v). Para la preparación de células se utilizó el método del aplastamiento (Östergren y Heneen, 1962). Las anteras se extrajeron con una pinza de disección y se colocaron en un portaobjetos. Se le agregó una gota del colorante carmín al $1 \%$, preparado con ácido acético al $45 \%$ para teñir los cromosomas. Con la aguja curva se presionó sobre las anteras para extraer las células, sobre las que se colocó un cubreobjetos. Se calentó por un minuto para posteriormente proceder suavemente al aplastado sobre un papel filtro y finalmente observar con un aumento de 1000x en un microscopio de luz visible. Con base en la calidad de las preparaciones se seleccionaron 53 células de tres plantas obtenidas de los cruzamientos de la línea HA 89 con tres progenitores masculinos individuales de $T$. rotundifolia etiquetados como TRG, TRI y TRK, y de los cuales toman sus nombres las tres plantas híbridas utilizadas.

El estudio de comportamiento meiótico se llevó a cabo en 32 células de la planta híbrida TRG, 8 de TRI y 13 de TRK. 
Los análisis cromosómicos se realizaron en las etapas de diacinesis, metafase I y anafase I. Las irregularidades cromosómicas se clasificaron en seis tipos: univalentes, multivalentes, cromosomas rezagados, cromosomas adelantados, puentes sin fragmentos y díadas desbalanceadas. Los cromosomas no apareados en diacinesis, o los mismos fuera de la placa ecuatorial en la metafase I, se definieron como univalentes. Los cromosomas rezagados y adelantados se definieron solamente para anafase I, en el sentido de su retraso o adelanto en la migración a los polos, evidencia de asincronía en la segregación.

\section{RESULTADOS Y DISCUSIÓN}

El apareamiento cromosómico en la meiosis de los híbridos fue irregular en las 53 células. Se observaron univalentes, multivalentes, cromosomas rezagados, cromosomas adelantados y puentes, así como díadas desbalanceadas en anafase tardía (Figura 1). En el Cuadro 1 se anotan los conteos de células para cada tipo de irregularidad meiótica encontrada en cada cruzamiento. Se observaron diferentes tipos de irregularidades en los meiocitos.

Para el grupo híbrido TRG, las ocho diacinesis presentaron univalentes o multivalentes, con un total de siete casos para cada una de esas irregularidades. En metafase I las ocho células presentaron univalentes, y siete de ellas multivalentes. En anafase I se observaron 16 células, en las cuales predominaron los desbalances cromosómicos (12 casos) y los cromosomas rezagados (10 casos). Solamente dos células exhibieron cromosomas adelantados y dos mostraron puentes no observándose fragmentos.

En el grupo TRI las cuatro células metafásicas presentaron cromosomas univalentes, y dos de ellas mostraron también multivalentes. Tres anafases presentaron cromosomas rezagados y dos anafases tardías presentaron díadas desbalanceadas. No se observaron puentes.
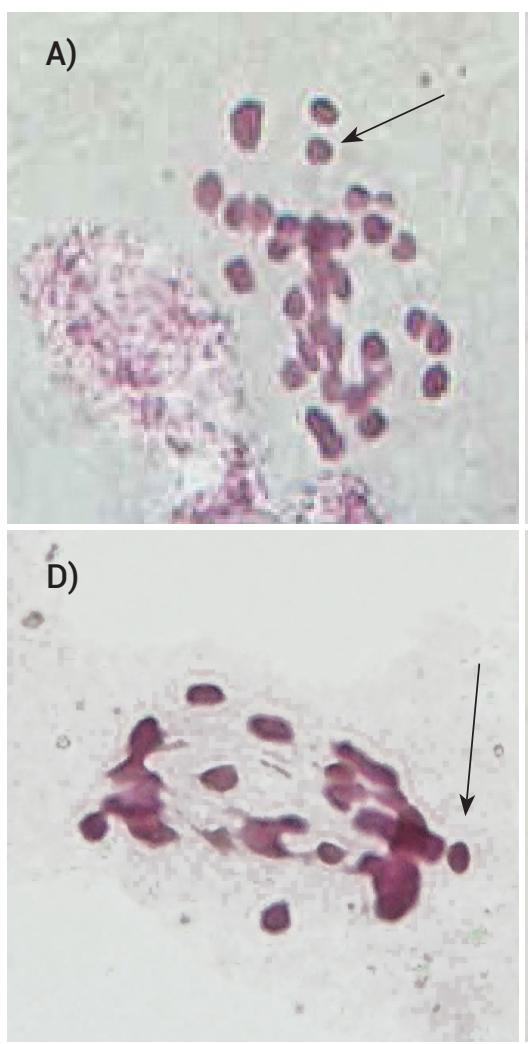

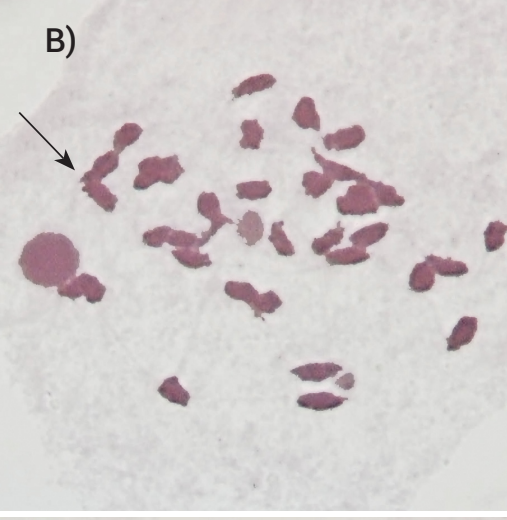

E)

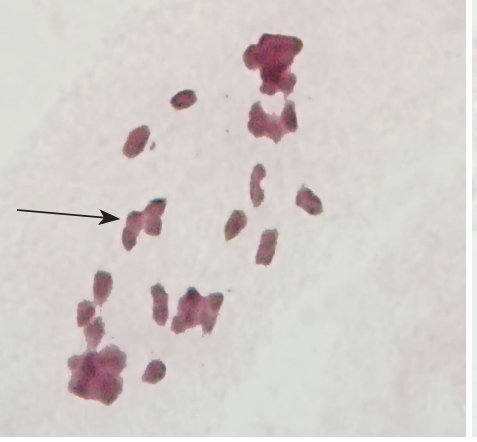

C)

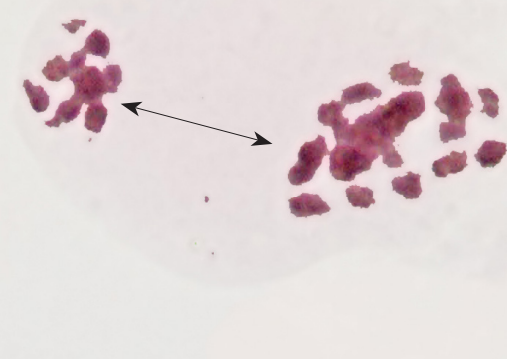

F)

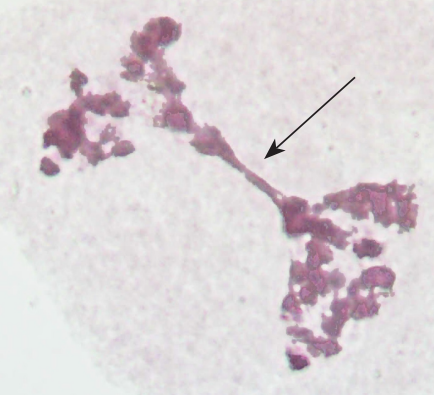

Figura 1. Irregularidades meióticas en plantas híbridas $H$. annuus $\times T$. rotundifolia. En cada célula hay un ejemplo señalado por una flecha A) Univalente en diacinesis-metafase I; B) Multivalente en diacinesis; C) Segregación desbalanceada (o díada desbalanceada) en anafase I tardía; D) Cromosoma adelantado en anafase I; E) Cromosoma rezagado en anafase I; F) Puente cromosómico (sin fragmento) en anafase I. 
Cuadro 1. Número de células meióticas con irregularidades meióticas en cruzamientos intergenéricos $H$. annuus $\mathbf{x} T$. rotundifolia. Los nombres de los cruzamientos están codificados con el identificador del progenitor femenino individual.

\begin{tabular}{llccccccc}
\hline \multirow{2}{*}{ Cruzamiento } & Fase & Univalentes & Multivalentes & $\begin{array}{c}\text { Díadas } \\
\text { desbalanceadas }\end{array}$ & Adelantados & Rezagados & Puentes & Totales \\
\hline TRG & Diacinesis & 7 & 7 & NA & NA & NA & NA & 8 \\
& Metafase I & 8 & 7 & NA & NA & NA & NA & 8 \\
\multirow{4}{*}{ TRI } & Anafase I & NA & NA & 12 & 2 & 10 & 2 & 16 \\
& Metafase I & 4 & 2 & NA & NA & NA & NA & 4 \\
TRK & Anafase I & NA & NA & 2 & 0 & 3 & 0 & 4 \\
& Diacinesis & 3 & 3 & NA & NA & NA & NA & 3 \\
& Metafase I & 1 & 1 & NA & NA & NA & NA & 1 \\
& Anafase I & NA & NA & 8 & 1 & 2 & 4 & 9 \\
\hline
\end{tabular}

NA = No aplica.

Para el grupo TRK se observaron tres células en diacinesis, todas con univalentes y multivalentes. En metafase I solamente se logró analizar una célula, la cual presentó tanto univalentes como multivalentes. Se analizaron nueve células en anafase I, de las cuales ocho tardías presentaron díadas desbalanceadas, y cuatro tempranas exhibieron puentes. Solamente se observaron dos células con cromosomas rezagados y una con cromosomas adelantados.

De lo anterior se desprende que ocurre una falla generalizada en el apareamiento meiótico, ya que no se observó un solo caso con división normal. La alta presencia de univalentes incide en anafases desbalanceadas, ya que originan segregación aleatoria de los cromosomas no apareados. Esto seguramente causa la formación de gametos con números cromosómicos irregulares, que impiden su fertilidad, tanto masculina como femenina.

Las irregularidades cromosómicas observadas en cada etapa no son independientes, sino que demuestran la falta de homología entre los juegos parentales de cromosomas. La falta de homología origina fallas en los entrecruzamientos y en el establecimiento de quiasmas. Ya que una función de éstos es orientar a los cromosomas en metafase I (Carpenter, 1994), las irregularidades en los mecanismos de su establecimiento y mantenimiento, originan errores en la segregación, que desembocan en la producción de gametos desbalanceados (Reyes-Valdés et al., 1996; Petronczki et al., 2003).

Irregularidades cromosómicas similares se han detectado en cruzamientos de $\mathrm{H}$. annuus con $\mathrm{H}$. tuberosus, $\mathrm{H}$. mollis, H. salicifolius y H. maximiliani (Atlagic et al., 1993; 1995); y en cruzamientos de $H$. annuus cultivado con $H$. annuus silvestre, $\mathrm{H}$. praecox y $\mathrm{H}$. argophyllus (Dolgova et al., 2007).
Las diferencias en los rearreglos cromosómicos entre las especies parentales tienen un fuerte efecto en el aislamiento reproductivo post-cigótico en las plantas, ya que sus efectos en la meiosis pueden afectar la fertilidad de los híbridos. Los tipos de rearreglo que más comúnmente dan lugar a dicho aislamiento reproductivo son las inversiones y las translocaciones (Baack et al., 2015). Por tanto, es verosímil que la presencia de translocaciones e inversiones contrastantes entre $H$. annuus y $T$. rotundifolia (al menos para Ac. 26), sea la causa de las fallas de apareamiento cromosómico que condujeron a la esterilidad híbrida de las plantas obtenidas por Luévanos-Escareño et al. (2010). Las anomalías en el apareamiento cromosómico son el resultado de la evolución divergente entre las dos especies, que han acumulado diferencias cromosómicas que alteran la homología.

Las dificultades para obtener híbridos intergenéricos fértiles podrían ser superadas con algunas técnicas de cultivo de tejidos como el rescate de embriones (Gómez-Martínez et al., 2010) y la duplicación del complemento cromosómico con colchicina. Sin embargo, los anfidiploides estables en este grupo de plantas son raros y exhiben baja fertilidad (Van Tassel, 2013).

\section{CONCLUSIONES}

Las irregularidades en el apareamiento meiótico que se observan en este estudio indican que los genomas parentales del híbrido integenérico $H$. annuus HA $89 \times$ T. rotundifolia Ac 26 no son compatibles en la meiosis, lo cual impide una segregación cromosómica balanceada y no permite la formación de gametos viables. Esto resulta en una barrera a la reproducción sexual de los híbridos. Estas diferencias impactan en gran medida el proceso meiótico, ya que no se observaron células con apareamiento normal. 


\section{AGRADECIMIENTOS}

Este trabajo fue financiado a través del proyecto CONACYT-SAGARPA 11971 y por la Universidad Autónoma Agraria Antonio Narro.

\section{BIBLIOGRAFÍA}

Atlagic J., B. Dozet and D. Skoric (1993) Meiosis and pollen viability in $\mathrm{He}-$ lianthus tuberosus $\mathrm{L}$. and its hybrids with cultivated sunflower. Plant Breeding 111:318-324.

Atlagic J., B. Dozet and D. Skoric (1995) Meiosis and pollen grain viability in Helianthus mollis, H. salicifolius, H. maximiliani and their $F$, hybrids with cultivated sunflower. Euphytica 81:259-263.

Baack E., M. C. Melo, L. H. Riesebergand and D. Ortiz-Barrientos (2015) The origins of reproductive isolation in plants. New Phytologist 207:968-984

Carpenter A. T. (1994) Chiasma function. Cell 77:959-962.

Christov M. (2013) Contribution to interspecific and intergeneric hybridization to sunflower breeding. Helia 36:1-18.

Cristov M. and I. Panayotov (1991) Hybrids between the genera Helianthus and Tithonia and their study. Helia 14:27-34.

Dolgova T. A., L. L. Yushkina and V. N. Popov (2007) Cytogenetic study of F interspecific hybrids of the section Helianthus. Helia 30:51-60.

Encheva J., H. Köhler, M. Christov, P. Shindrova, V. Encheva and W. Friedt (2014) New sunflower ( $H$. annuus L.) lines as results of interspecific and intergeneric hybridization and application of method of direct organogenesis in $\mathrm{F}_{1}$ immature embryo. Bulgarian Journal of Agricultural Science 20:1444-1449.

Gómez-Martínez M., M. H. Reyes-Valdés, J. M. Martínez-Reyna, L. EscobedoBocardo y H. T. García-Osuna (2010) Rescate de embriones en híbridos intergenéricos Helianthus annuusxTithonia rotundifolia. Acta Botánica Mexicana 93:111-119.

Jan C. C. (1997) Cytology and interspecific hybridization. In: Sunflower Technology and Production. A. A. Schneiter (ed.). ASA-CSSASSSA. pp: 497-558.

Luévanos-Escareño M. P., M. H. Reyes-Valdés, J. A. Villarreal-Quintanilla y R. Rodríguez-Herrera(2010) Obtención de híbridos intergenéricos Helianthus annuus $x$ Tithonia rotundifolia y su análisis morfoló- gico y molecular. Acta Botánica Mexicana 90:105-118.

Muoghalu J. I. and D. K. Chuba (2005) Seed germination and reproductive strategies of Tithonia diversifolia (HEMSL.) gray and Tithonia rotundifolia (P. M) Blake. Applied Ecology and Environmental Research 3:39-46.

Östergren G. and W. K. Heneen (1962) A squash technique for chromosome morphological studies. Hereditas 48:332-341.

Petronczki M., M. F. Siomos and K. Nasmyth (2003) Un menage a quatre: the molecular biology of chromosome segregation in meiosis. Cell 112:423-440.

Reyes-Valdés M. H., M. Gómez-Martínez, O. Martínez and F. Hernández Godínez (2005) Intergeneric hybrid between cultivated sunflower (Helianthus annuus L.) and Tithonia rotundifolia (Mill.) Blake. Helia 28:61-68

Reyes-Valdés M. H, Y. Ji, C. F. Crane, M. N. Islam-Faridi, H. J. Price, D. M. Stelly and J. F. Taylor (1996) ISH-facilitated analysis of meiotic bivalent pairing. Genome 39:784-792

Reyes-Valdés M. H., J. A. Villarreal-Quintanilla, F. J. Santana-Michel y J. Salmerón-Erdosay (2007) Localización y recolección de Tithonia rotundifolia en el suroeste de México. In: Proceedings of the Interamerican Society for Tropical Horticulture. Volume 51. N. Ledesma, C Balerdi, J Crane, F Mangan and N Maciel (eds.) 53nd Annual Meeting Interamerican Society for Tropical Horticulture. Morelia, Mich. 7-12 Oct. Interamerican Society for Tropical Horticulture. pp:274-278.

Seiler G. J. and L. H. Rieseberg (1997) Systematics, origin, and germoplasm resources of the wild and domesticated sunflower. In: Sunflower Technology and Production. Agronomy Monograph \# 35. A. A. Schneiter (ed.). ASA-CSSA-SSSA. pp:21-65

Tovar-Sánchez E., F. Rodríguez-Carmona, V. Aguilar-Mendiola, P. MussaliGalante, A. López-Caamal and L. Valencia-Cuevas (2012) Molecular evidence of hybridization in two native invasive species: Tithonia tubaeformis and T. rotundifolia (asteraceae) in Mexico. Plant Systematics and Evolution 298:1947-1959.

Van Tassel D. L., S. R. Asselin, S. A. Cox, G. Sideli and D. J. Cattan (2013) Evaluating perennial candidates for domestication: lessons from wild sunflower relatives. In: Perennial Crops for Food Security. Proceedings of the FAO Expert Workshop. C. Batello, L. Wade, S. Cox, N. Pagna, A. Bozzini and J. Choptiany (eds.). FAO Expert Workshop. Rome, Italy. 28-30 Aug. FAO. Rome, Italy. pp:112140 